\title{
Prediction of Cross-Tension Strength of Self-Piercing Riveted Joints Using Finite Element Simulation and XGBoost Algorithm
}

\author{
Jianping Lin ${ }^{1}$, Chengwei Qi ${ }^{1}$, Hailang Wan ${ }^{1}$, Junying Min ${ }^{1 *} \mathbb{D}$, Jiajie Chen ${ }^{2}$, Kai Zhang ${ }^{1}$ and Li Zhang ${ }^{2}$
}

\begin{abstract}
Self-piercing riveting (SPR) has been widely used in automobile industry, and the strength prediction of SPR joints always attracts the attention of researchers. In this work, a prediction method of the cross-tension strength of SPR joints was proposed on the basis of finite element (FE) simulation and extreme gradient boosting decision tree (XGBoost) algorithm. An FE model of SPR process was established to simulate the plastic deformations of rivet and substrate materials and verified in terms of cross-sectional dimensions of SPR joints. The residual mechanical field from SPR process simulation was imported into a 2D FE model for the cross-tension testing simulation of SPR joints, and cross-tension strengths from FE simulation show a good consistence with the experiment result. Based on the verified FE model, the mechanical properties and thickness of substrate materials were varied and then used for FE simulation to obtain cross-tension strengths of a number of SPR joints, which were used to train the regression model based on the XGBoost algorithm in order to achieve prediction for cross-tension strength of SPR joints. Results show that the cross-tension strengths of SPR steel/aluminum joints could be successfully predicted by the XGBoost regression model with a respective error less than $7.6 \%$ compared to experimental values.
\end{abstract}

Keywords: Self-piercing riveting, Joint strength, Cross-tension, Finite element modeling, Machine learning

\section{Introduction}

Reduction in weight of automobile parts is a prevailing trend in automobile industry. An effective method for lightweight is using high strength steel and aluminium alloy as materials of automobile parts [[1]]. There are several researches on the development of lightweight materials [[2]], advanced material testing [[3]], accurate simulation of forming process [[4]], and so on. With regard to the mechanical joining process of high strength steels and aluminium alloys, self-piercing riveting (SPR) is a suitable method [[5]] due to the advantages of environmental friendliness, high joint strength and stiffness [[6]], easy access to visually inspect the joints and

\footnotetext{
${ }^{*}$ Correspondence: Junying.Min@tongji.edu.cn

${ }^{1}$ School of Mechanical Engineering, Tongji University, Shanghai 201804, China

Full list of author information is available at the end of the article
}

possibility of manual application [[7]]. Nowadays, SPR has been widely used by major automotive manufacturers, and some scholars even use electromagnetic-SPR to widen its application [[8], [9]].

Joint strength is the primary factor to evaluate SPR joint quality. The strengths of SPR joints are generally obtained through conventional mechanical tests, which is costly and time-consuming. To solve this problem, Sun and Khaleel [[10]] proposed an analytical model to predict the static strength based on some cross-sectional dimensions of SPR joints and characteristics of substrates. Nine different cases consisting of aluminium and steel materials with various thicknesses and strength grades were examined to validate the model. Later, an empirical equation without using cross-sectional dimensions of SPR joints was established by Haque et al. [[11]]. The empirical equation can achieve the prediction of cross-sectional dimensions and lap-shear strengths directly based on the 
force-displacement curve of SPR process, and the predicted strengths of SPR joints matched the experimental strengths reasonably. Xie et al. [[12]] established an empirical model to predict SPR steel/steel joints strength under all kinds of failure modes. Considering the strength reduction in the case of group rivets, Yan et al. [[13]] proposed a design method based on the model of transmission dynamics of infectious diseases to estimate the shear strength of the rivet connections. The nominal shear strength of SPR joint could be appropriately predicted. Ma et al. [[14]] built a model to predict the lap-shear strength of SPR joints according to top sheet thickness and undercut dimension, the model was only able to predict the lap-shear strength of SPR joints made of specific materials (AA6061-T6 and mild steel CR4) with various thickness. Although analytical or empirical model can predict the strength of SPR joints, their applications were mostly limited by the cost of the experiments required to calibrate the model, complex calculations, and poor versatility, etc.

In general, a large amount of experiment results is required to verify the prediction accuracy of theoretical model, however, using FE method to obtain joint strength is more economical than experiment. Several researches report that the mechanical behavior of SPR joints can be well simulated by FE model. Westerberg [[15]] used ABAQUS/Explicit to simulate the T-peeling test of SPR joint, the cross-sectional dimensions of experimental joints were used to build the simulation model. Various test speeds were used in the simulation, and the results showed that the mechanical performance was similar at a test speed of 1,10 and $25 \mathrm{~m} / \mathrm{s}$, but the maximum load, energy absorption and failure displacement of SPR joint were higher when tested at a higher speed of $100 \mathrm{~m} / \mathrm{s}$. Porcaro et al. [[16]] studied the mechanical behavior of SPR joints through LS-DYNA. A 2D model was established for SPR process simulation, and then the simulation results of residual stress and strain from the SPR process model were imported into a 3D model for the simulation of joint strength. Bouchard et al. [[17]] compared the predicted shear strengths obtained from the simulation model with and without the initial mechanical fields caused by SPR process, their results indicated that the prediction results obtained from the model considering initial mechanical fields matched better with the experiment results. Result from Moraes et al. [[18]] demonstrated that residual stresses and plastic strain from the SPR process dramatically changed the quasi-static and fatigue behaviors of SPR joint. As reviewed above, an FE model considering the plastic strain and residual stress induced from SPR process can effectively help the prediction of SPR joint strengths, but it is still a timeconsuming method.
The engineering application of machine learning method is an attractive approach lately. The advantage of using machine learning method to build predict model for the strength of SPR joints is that the model is scalable, in other words, the prediction accuracy can be improved with the increasing of used training data. Chen et al. [[19]] applied least squares support vector machine (LSSVM) to predict grinding chatter and reached a prediction accuracy rate of $96 \%$. Shao et al. [[20]] extract feature from motors' original signals and used deep belief networks (DBN) to achieve automated and intelligent fault diagnosis for induction motors. Fujishima et al. [[21]] proposed a novel compensation method using deep learning algorithm to compensate the thermal deformation in machine tool structure. Postel et al. [[22]] designed an approach for the inverse identification of parameters during cutting operation to predict. Yavuz et al. [[23]] developed an artificial neural network (ANN) model to estimate the shear capacities of the FRP-strengthened reinforced concrete beams, they concluded that the ANN model had a better prediction accuracy than existing building code approaches. Qin et al. [[24]] used deep-learning technology to establish an end-to end relationship between cross-sectional SEM images of cement backfill beam (CPB) and its mechanical strength, a convolutional neural network was used to predict the mechanical strength of CPB based on the features extracted from the cross-sectional SEM images. But prediction models using machine learning algorithms usually require huge data sets to achieve accurate predictions, and it is not economical to obtain data sets through experiments. To solve this problem, Liu et al. [[25]] developed a model based on FE model and ANN to predict and compensate force-induced deformation of machine tools for dual-machine-based riveting system.

After comparing the above methods, it is deducible that using machine learning method to predict the strength of SPR joint based on FE model data is effective and time-saving. Therefore, a model was developed to predict the cross-tension strength of SPR joints based on FE model data and XGBoost regression model in this work. The structure of the work is organized as follows: Section 2 introduces the SPR process and mechanical test experiment. In Section 3, the detail of FE modeling and model validation are provided. In Section 4, the establishment and analysis of the strength prediction model are discussed in details, and the conclusions and outlook are summarized in Section 5.

\section{Experimental Details}

\subsection{Materials}

The substrate materials used in this study were coldrolled zinc-galvanized steel CR590 (1.1 mm in thickness), 
aluminum alloy S-6000-IH (1.2 $\mathrm{mm}$ in thickness), aluminum alloy AA6022-T4 (2.0 mm in thickness) and aluminum alloy AA5754-O (2.0 $\mathrm{mm}$ in thickness), respectively. The four materials used in this work have a wide application on body-in-white (BIW) of automotive. CR590 steel, a dual phase steel with a strength grade of $600 \mathrm{MPa}$, is widely used in automobile's A pillar, B pillar and their reinforcement plates. The $\mathrm{Al}$ alloys adopted in this work are widely used in inner and outer panels of car body. SPR is an effective method to join dissimilar materials, e.g., steel and $\mathrm{Al}$ alloy. The rivet material was $37 \mathrm{Cr} 4$ steel. Table 1 summarizes the mechanical properties of experimental materials provided by suppliers. In order to obtain the specific material properties of the substrate materials used in SPR modeling, quasi-static uniaxial tensile tests were performed with the aid of digital image correlation (DIC) method according to the GB/T228.12010 standard. The true stress-strain curves derived from uniaxial tensile test results were extrapolated using the Hollomon model (Eq. (1)), and the fitting parameters of substrate materials are summarized in Table 2. Due to the small volume and hollow structure of the rivet, compression tests with the wire rivet material along longitudinal direction were conducted to obtain its true stress-strain curve. Then, the acquired material property curve was fitted using the Hockett-sherby model (Eq. (2)) [[26]] with fitting parameters of $B=1555.3 \mathrm{MPa}, A=1762.3$ $\mathrm{MPa}, m=51.2$ and $n=0.80$ :

$$
\begin{aligned}
& \sigma=K \cdot \varepsilon^{n}, \\
& \sigma=B-(B-A) \cdot e^{-m \varepsilon_{p}^{n}} .
\end{aligned}
$$

\subsection{SPR Joint Fabrication}

Results from Abe et al. [[26]] showed that the SPR steel/ aluminum joints possess higher strength when steel was used as the top sheet. Therefore, in this work, the steel was used as the top sheet and the aluminum alloy used as the bottom sheet. The cross-sectional geometries of Zinc-coated $37 \mathrm{Cr} 4$ steel rivets with countersunk head and SPR die are illustrated in Figure 1. All SPR joints

Table 1 Mechanical properties of the substrate and rivet materials

\begin{tabular}{lcll}
\hline Material grade & $\begin{array}{l}\text { Young's } \\
\text { modulus (GPa) }\end{array}$ & $\begin{array}{l}\text { Ultimate tensile } \\
\text { stress (MPa) }\end{array}$ & Yield stress (MPa) \\
\hline CR590 & 198 & 621 & 369 \\
S-6000-IH & 62 & 225 & 120 \\
AA6022-T4 & 62 & 262 & 159 \\
AA5754-O & 70 & 227 & 100 \\
37Cr4 (rivet) & 210 & 1700 & 1500 \\
\hline
\end{tabular}

Table 2 Fitting parameters of Hollomon model for substrate materials

\begin{tabular}{lcccc}
\hline Material grade & CR590 & S-6000-IH & AA6022-T4 & AA5754-O \\
\hline K(MPa) & 999.4 & 390.0 & 449.6 & 448.5 \\
$n$ & 0.17 & 0.21 & 0.18 & 0.29 \\
\hline
\end{tabular}

were produced with the EPRESS Ltd. servo driven riveting equipment using a concave die as shown in Figure 1. The riveting speed was set to $1 \mathrm{~mm} / \mathrm{s}$ and a riveting force of $50 \mathrm{kN}$ (maximum force on the punch) was applied.

According to Zhu et al. [[27]], cross-tension joints for SPR are fabricated as shown in Figure 2. Three material combinations (A to $\mathrm{C}$ listed in Table 3) were considered in this study (referred to as SPR-A, SPR-B, SPR-C joints), and three joints were produced for each combination (Figure 2a). A customized fixture was used to ensure that the peeling force is along the central axis of the joint during the cross-tension test (as presented in Figure 2b), and the cross-tension tests of SPR joints were performed with a cross-head speed of $10 \mathrm{~mm} / \mathrm{min}$ on a universal testing machine MTS E45.105. The Keyence VHX-6000 digital microscope system was then used to observe the crosssectional morphology and dimensions of SPR joint.

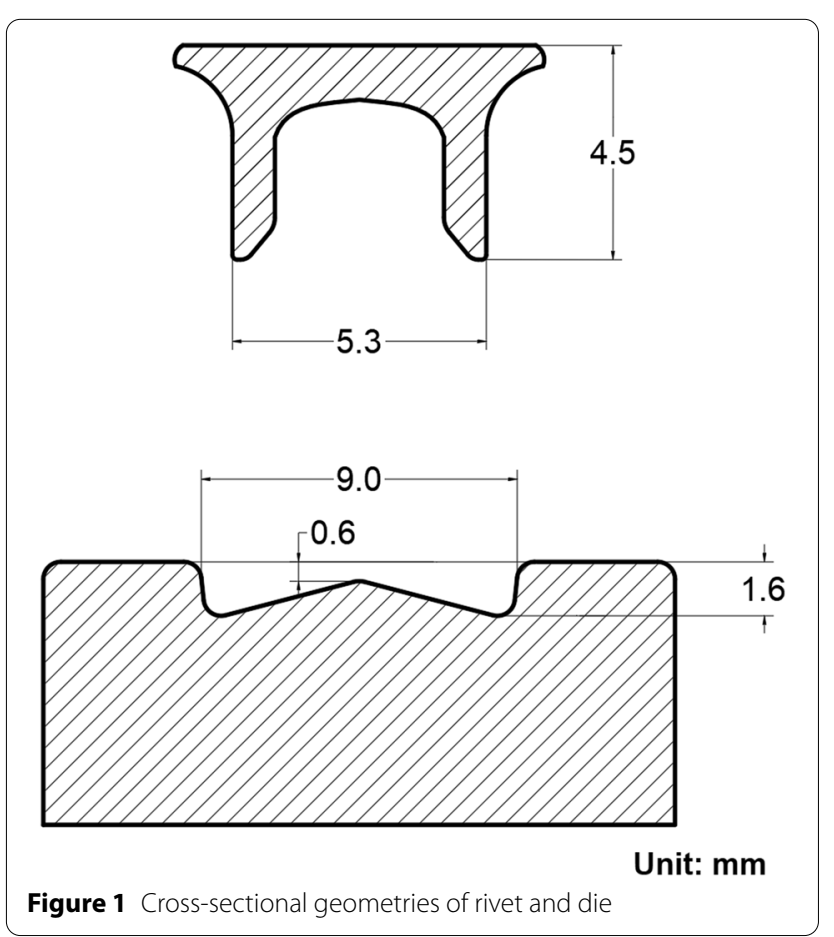




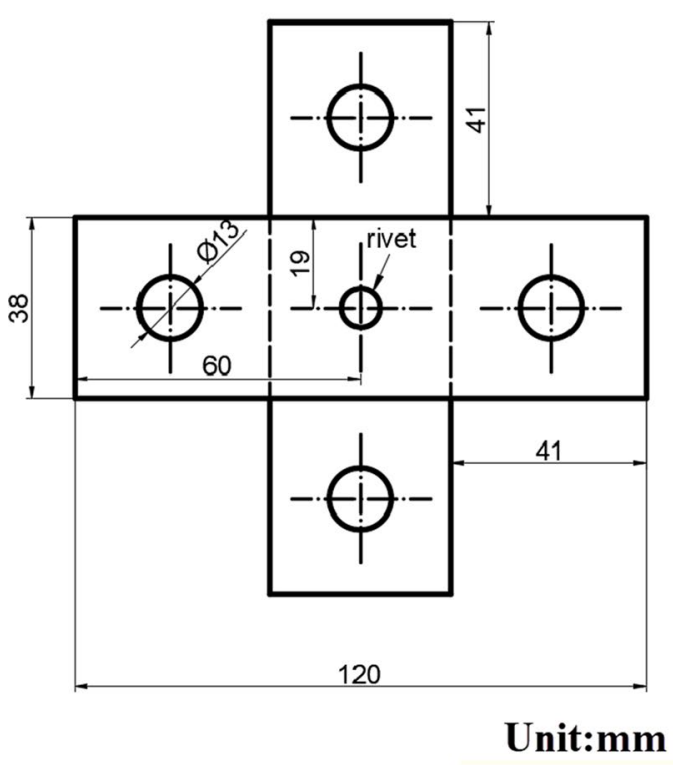

a

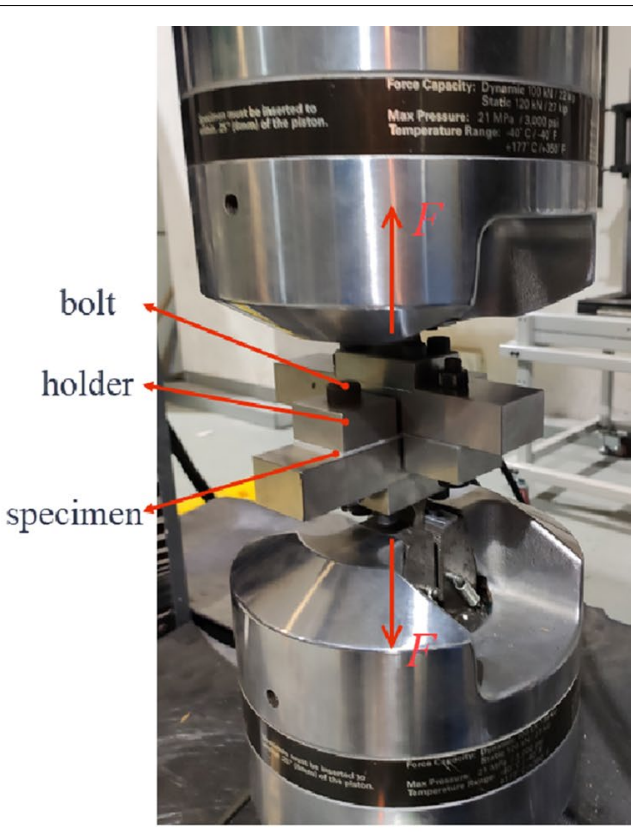

b

Figure 2 Schematic dimension of specimen and optical image of test setup (a) dimensions of cross-tension joint, (b) cross-tension test setup

Table 3 Summary of joining combinations for cross-tension strength analysis

\begin{tabular}{lllc}
\hline Combination & A & B & C \\
\hline $\begin{array}{l}\text { Top sheet material (thick- } \\
\text { ness) }\end{array}$ & & CR590 (1.1 mm) & \\
$\begin{array}{l}\text { Bottom sheet material } \\
\text { (thickness) }\end{array}$ & S-6000-IH & AA6022-T4 (2.0 mm) & AA5754- \\
& $(1.2$ & & O (2.0 \\
& $\mathrm{mm})$ & $\mathrm{mm})$ \\
\hline
\end{tabular}

\section{Numerical Simulation}

\subsection{Finite Element Modeling of SPR Process}

Numerical simulation of the SPR process and SPR joints' cross-tension process was performed using the finite element software Simufact. forming. It includes the implicit MSC Mar solver for non-linear applications and has SPR simulation module. In this study, the punch, die and blank holder were set as rigid bodies, and the rivet material and the substrate materials were defined as elastoplastic materials in the numerical simulation. As shown in Figure 3, two rigid parts were added to facilitate subsequent simulation of cross-tension test. The punch speed was set to $1 \mathrm{~mm} / \mathrm{s}$, which is consistent with the actual riveting speed in the riveting experiments, and the clamping force of $5 \mathrm{kN}$ was applied to the blank holder through a compressed spring.

The Coulomb friction law was used in the SPR process simulation. The friction coefficient between

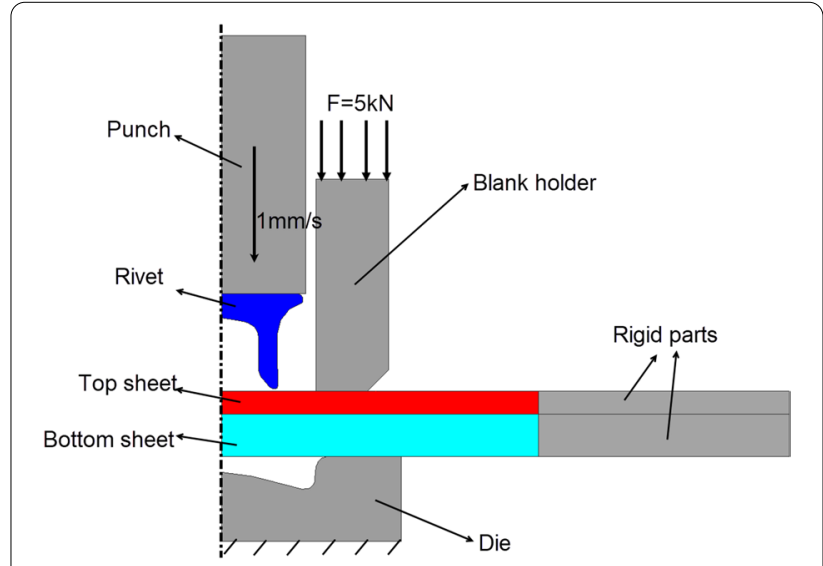

Figure 3 Boundary condition of SPR process simulation

bottom sheet and die was set to 0.4 , while the friction coefficients elsewhere were set to 0.15 [[28]]. A 2D axisymmetric model was used for SPR process simulation to save calculation time. The rivet was meshed using 4-nodes quadrilateral elements with a mesh size of $0.15 \mathrm{~mm} \times 0.15 \mathrm{~mm}$, while the top and bottom sheets were meshed using 4-nodes advancing front quad elements with a mesh size of $0.2 \mathrm{~mm} \times 0.2 \mathrm{~mm}$. SPR of two sheet combinations was modelled: $1.1 \mathrm{~mm} / 1.2 \mathrm{~mm}$ and $1.1 \mathrm{~mm} / 2.0 \mathrm{~mm}$. As shown in Figure 4, mesh refinement boxes were applied to the rivet tip (solid rectangle 


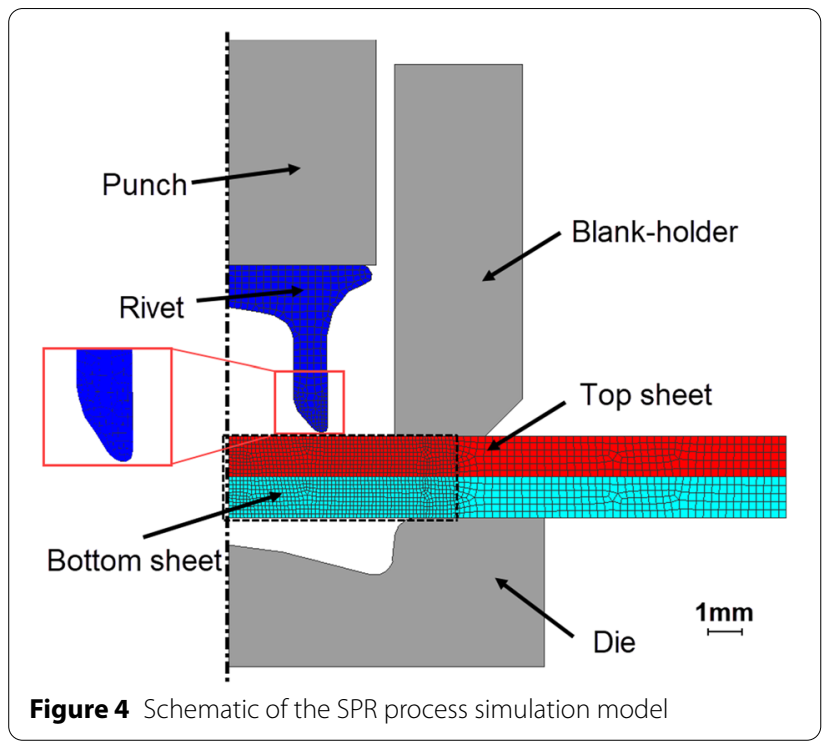

in Figure 4) and the central deformation areas of the top and bottom sheets (dashed rectangle in Figure 4) to distribute fine meshes and improve the prediction accuracy without remarkably increasing calculation time. Due to large deformations occurring at the cutting zone in front of the rivet tip, mesh rezoning algorithm was necessary. The remeshed elements in the cutting zone become smaller until the minimum blank thickness is reached to achieve accurate geometrical cutting. The minium mesh size of top and bottom sheet during remeshing was set to be $0.025 \mathrm{~mm} \times 0.025 \mathrm{~mm}$.

A geometrical criterion based on a separate thickness (i.e., $0.05 \mathrm{~mm}$ ) was implemented to define the finish of rivet piercing through the top sheet (i.e., CR590 steel) in the simulation model. As illustrated in Figure 5, the top sheet is assumed as completely pierced by the rivet when its minimum remaining thickness is less than the separate thickness. The riveting process was controlled by the riveting force and were terminated when the riveting force reached $50 \mathrm{kN}$. After that the punch and blank holder were moved up to release the joint from the die, then the spring back of SPR joints were calculated.

\subsection{Finite Element Modeling of Cross-Tension Testing of SPR Joints}

The model of cross-tension test in this study was established based on the result of SPR process simulation. It is assumed that the rivet periphery was under perfectly axisymmetric loading right before failure during cross-tension test [[10]]. Under this assumption, a 2D axisymmetric model can be used to simulate the crosstension process to save simulation time. The boundary

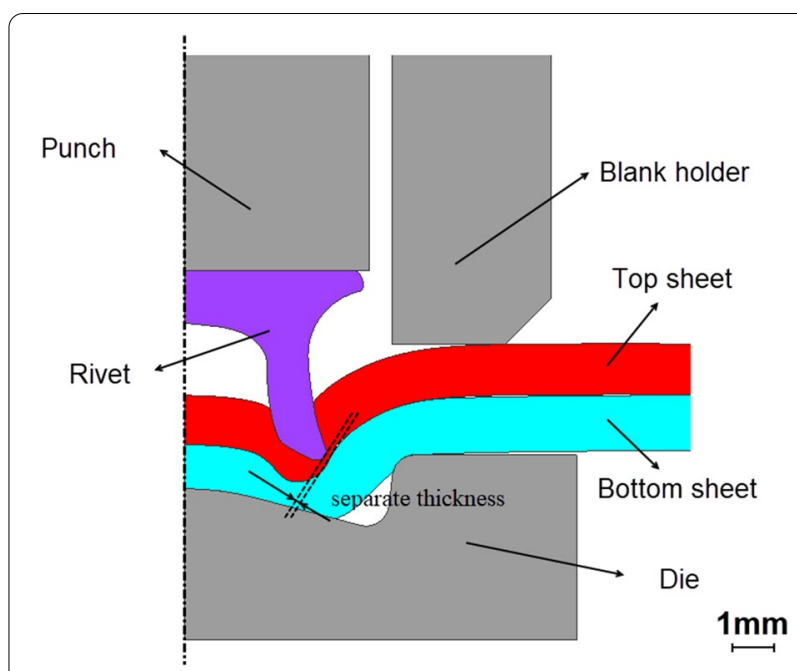

Figure 5 Illustration of the separate thickness for blanking of the top sheet

condition of 2D cross-tension test model is shown in Figure 6. The load was applied to a rigid part (grey part in Figure 6), and the loading speed was set to $10 \mathrm{~mm} /$ min for 2D cross-tension model. The coulomb friction coefficients at all contacts in the model were set to 0.15 .

\subsection{Validation}

To verify the established simulation model of SPR process, the cross-sectional morphologies and dimensions of SPR-A and SPR-B joints obtained from simulation were compared with those obtained from experiments. It can be seen from Figure 7 that the profiles obtained from simulations matched well with those from experiments. According to Haque's research [[29]], the undercut (UD) and the minimum remaining thickness (RT) of the bottom sheet are two key dimensions that reflect SPR joint quality. Therefore, the values of UD and RT were measured for joints obtained from experiment and simulation as summarized in Table 4. Good agreement between the simulations and the experiments indicates that the developed simulation model is capable of predicting the deformation of the rivet and sheets during SPR process.

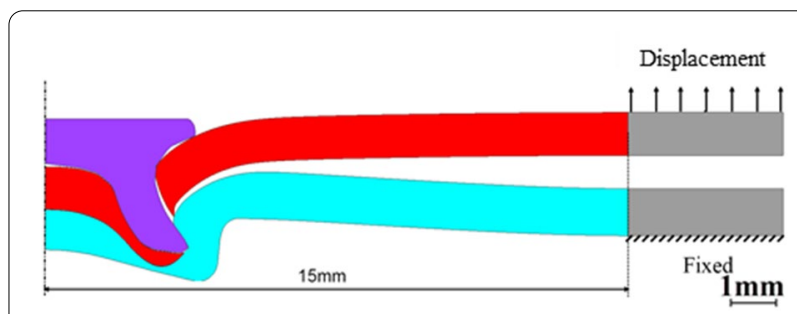

Figure 6 2D FE model of cross-tension test for SPR joint 


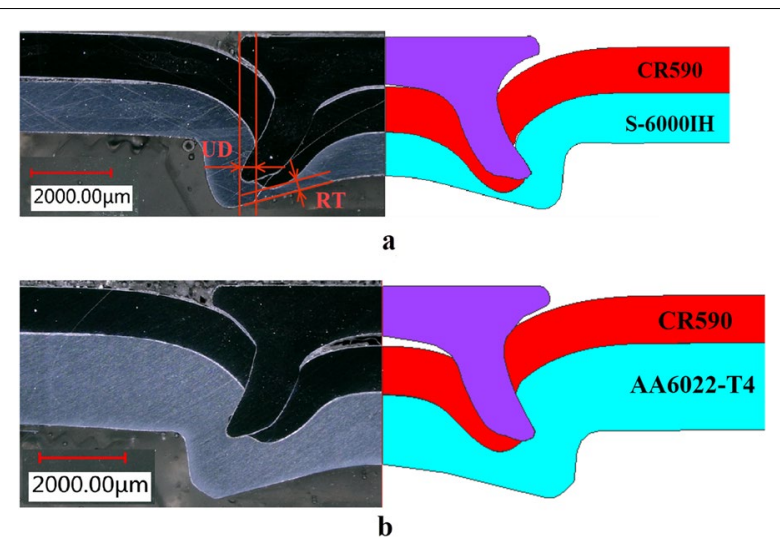

Figure 7 Comparison of the joint cross-sectional profiles from the simulations and experiments (a) SPR-A joint, (b) SPR-B joint

Figure 8 presents the comparisons of force-displacement curves between simulation and experiment. The strength obtained experimentally and numerically for SPR-A are $1.57 \pm 0.02 \mathrm{kN}$ and $1.61 \mathrm{kN}$ (with an error of $2.54 \%$ ), while for SPR-B $3.04 \pm$ are $0.02 \mathrm{kN}$ and 3.18 $\mathrm{kN}$ (with an error of $4.61 \%$ ), respectively. It can be observed that the strengths of SPR-A and SPR-B joints obtained from simulations were close to the experimental values, while a difference in the failure displacement was observed for both of SPR-A and SPR-B joints, which is attributed to the following two aspects: (1) To ensure that the specimen can be clamped smoothly, the diameter of mounting holes (i.e., 13 $\mathrm{mm}$ ) on the specimen are larger than that of used fixing bolts (i.e., $12 \mathrm{~mm}$ ), and the sliding gap is $\sim 1 \mathrm{~mm}$ for top and bottom specimens during cross-tension testing; therefore, a sliding displacement of $\sim 2 \mathrm{~mm}$ is generated during tensile testing, which does not occur in simulation (referred to Figure 9). (2) The deformable area of specimens in simulation model is set as a semicircle with a radius of $15 \mathrm{~mm}$ (referred to Figure 9), which is smaller than that in experiment (i.e., a square with a size of $38 \mathrm{~mm} \times 38 \mathrm{~mm}$ ), thus, a larger deformation displacement is generated for SPR joints during cross-tension test. However, this work mainly focuses on the joint strength, and a good agreement was found between FE model and experiment with respect to the cross-tension strength for SPR-A and
SPR-B joints (with only an error of $1.3 \%$ ). As a result, the established FE model is capable of predicting the cross-tension strength for SPR joints.

\section{Prediction of Cross-Tension Strength of SPR Joints Using XGBoost Regression Model}

\subsection{XGBoost Regression Model}

Theoretically, the traditional neural network method can achieve the regression through fitting of any non-linear function, but it has poor stability and highly depends on data quantity. XGBoost is a scalable end-to-end tree boosting algorithm with high stability and accuracy, which was recently proposed by Chen et al. [[30]]. It has strong fitting ability and is suitable for regression compared with tradition back-propagate algorithm. A regularization term was introduced into the objective function of XGBoost regression model to avoid the overfitting phenomenon that occurs in tradition decision tree algorithms. The Classification and Regression Trees was used as base learner. The overall objective function of XGBoost regression model is expressed by Eq. (3):

$$
\operatorname{Obj}(\theta)=L(\theta)+\Omega(\theta)+C .
$$

The parameters of XGBoost model can be divided into three categories: overall parameters, acceleration parameters and tuning parameters. For overall parameters, when the booster parameter is set to 'gblinear', the model is a linear model, and when the booster parameter is set to 'gbtree', the model is a tree-based model. In this work, the booster parameter was set to 'gbtree' to reflect the highly nonlinear relationship between the input data and output data. Among the acceleration parameters, there are many parameters that need to be adjusted. The eta value controls the robustness of the model, and the default value of 0.3 was used in this work. Other parameters are determined during training of the model.

\subsection{Data Set of the XGBoost Regression Model}

To acquire a sufficient number of data as input, the thickness and material properties ( $K$ and $n$ parameters in the Hollomon hardening law) of the bottom sheet were varied and then used for simulations. Six material properties with various $K$ and $n$ values were created for the bottom materials of SPR joints as listed in Table 5 and used for FE

Table 4 Comparison of the joint cross-sectional dimensions from the simulation and experiment

\begin{tabular}{llllcr}
\hline Joint & \multicolumn{2}{l}{ Undercut (UD, mm) } & & Error (\%) & \multicolumn{2}{c}{\begin{tabular}{c} 
Minimum remaining thickness (RT, mm) \\
\cline { 2 - 3 }
\end{tabular}} & FE model & Experiment & FE model \\
\hline SPR-A & 0.41 & 0.39 & 6.2 & 0.16 & 0.17 \\
SPR-B & 0.43 & 0.42 & 2.4 & 0.83 \\
\hline
\end{tabular}




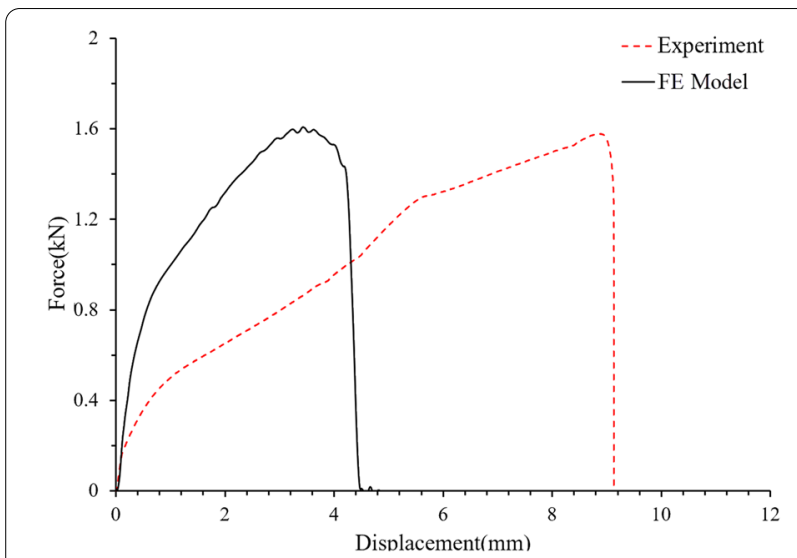

a

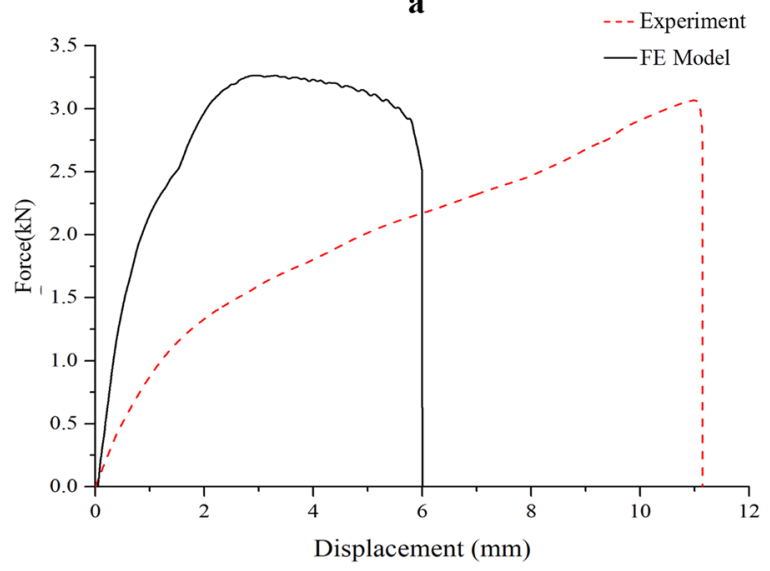

b

Figure 8 Comparison of force-displacement curve from FEM and experiment (a) SPR-A joint, (b) SPR-B joint model to acquire the cross-tension strengths. Only three kinds of thicknesses were selected for M3-M6 materials since the required amount of data has been achieved. Accordingly, a total of 48 cross-tension strengths of SPR joints were obtained and then used as the input data for XGBoost model.

\subsection{Prediction Model Development and Analysis}

The modeling steps of the XGBoost regression model are as follows [[30]]:

(1) Data processing. First, normalize the variables of $K$, $n$ and thickness to keep their values in similar magnitude levels. Then, the 48 base data sets of crosstension strengths were randomly divided into 34 training sets and 14 testing sets. Finally, input the base data sets into the XGBoost regression model.

(2) Determine parameters of XGBoost model by training data sets. 34 training data sets are input into the XGBoost regression model with some basic parameters to determine remaining parameters, and then calculate the prediction strengths for 34 training data sets. If the prediction error for training data sets was smaller than the target value, the XGBoost regression model is obtained, otherwise, the parameters of XGBoost regression model will be updated and then used to calculate the prediction strengths.

(3) Calculate the prediction error for testing data sets. After obtained the parameters of XGBoost regression model, the cross-tension strengths of SPR joints in testing data sets were predicted, and then

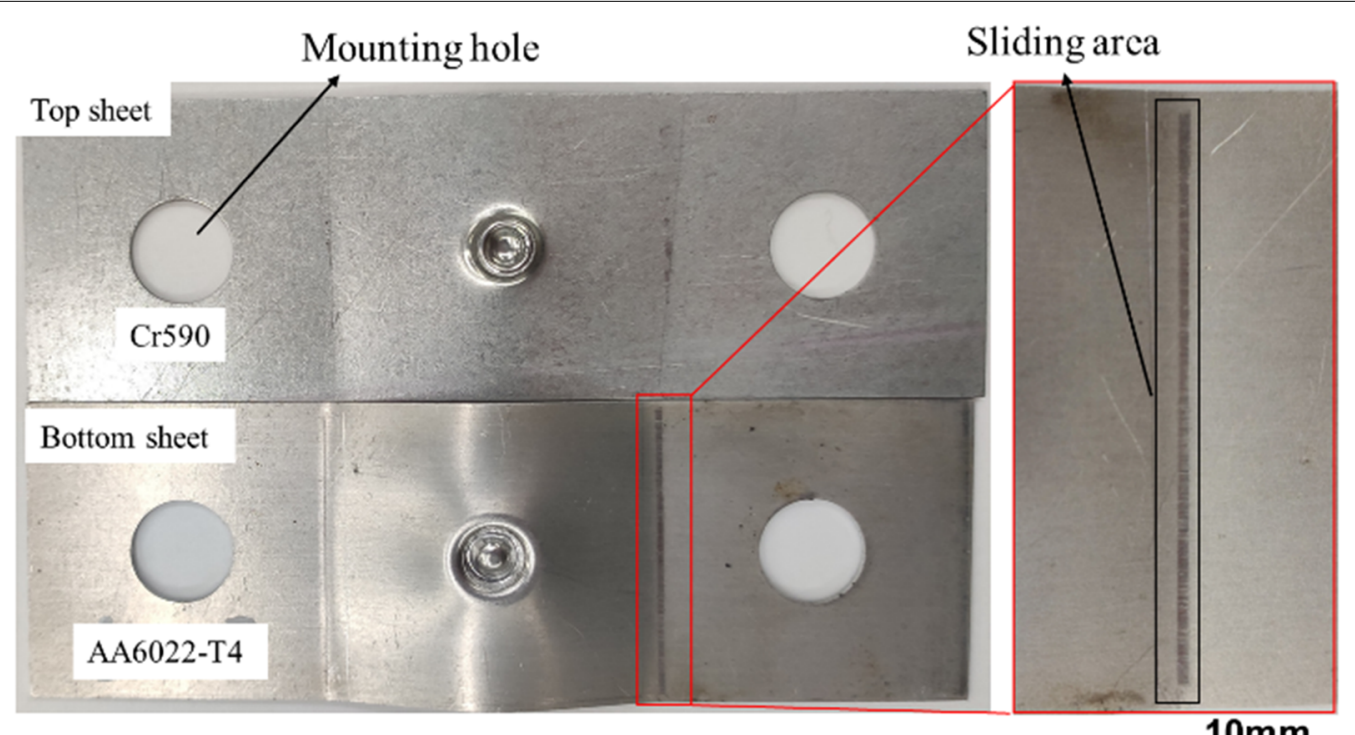

$10 \mathrm{~mm}$

Figure 9 Failure mode of SPR-B joint in cross-section test 
Table 5 Hollomon model parameters and thickness of bottom sheet material used in the simulation

\begin{tabular}{|c|c|c|c|}
\hline Bottom sheet material & $K(\mathrm{MPa})$ & $n$ & Bottom sheet thickness ( $\mathrm{mm}$ ) \\
\hline S-6000-IH & 390.0 & 0.21 & $\begin{array}{l}1.2,1.3,1.4,1.5,1.6,1.7,1.8 \\
1.9,2.0\end{array}$ \\
\hline AA6022-T4 & 449.6 & 0.18 & $\begin{array}{l}1.2,1.3,1.4,1.5,1.6,1.7,1.8 \\
1.9,2.0\end{array}$ \\
\hline M1 & 419.8 & 0.20 & $\begin{array}{l}1.2,1.3,1.4,1.5,1.6,1.7,1.8 \\
1.9,2.0\end{array}$ \\
\hline M2 & 510.0 & 0.16 & $\begin{array}{l}1.2,1.3,1.4,1.5,1.6,1.7,1.8 \\
1.9,2.0\end{array}$ \\
\hline M3 & 390.0 & 0.30 & $1.2,1.5,2.0$ \\
\hline M4 & 390.0 & 0.40 & $1.2,1.5,2.0$ \\
\hline M5 & 510.0 & 0.30 & $1.2,1.5,2.0$ \\
\hline M6 & 510.0 & 0.40 & $1.2,1.5,2.0$ \\
\hline
\end{tabular}

the prediction error was calculated to see whether the prediction accuracy is guaranteed.

(4) Cross-validation. Cross-validation is a model assessment technique and generally used to evaluate the prediction performance of a machine learning algorithm for new data sets that it has not been trained with. And 100 rounds of cross-validation were performed by using different seed parameters to acquire different random assignments of train- ing and testing data sets. The prediction error of each round of cross validation was calculated and the average prediction error (cross-validation error) was used as a performance indicator.

(5) Obtain the optimal parameters of XGBoost regression model. Output the optimal parameters once the cross-validation error reaches the target value, eventually, the desired XGBoost regression model are acquired. The establishing process of the prediction model is illustrated in Figure10.

After the training process was completed, testing set was used to verify the generalization capacity of the prediction model. The coefficients of determination $\left(R^{2}\right)$ for the training set and testing set were 0.9978 and 0.9677 , respectively, which indicates that the prediction results of the model were accurate. Figure 11 shows that the prediction results are consistent with input base data for the training set and testing set, and the percentage error of the training set and testing set is less than $1 \%$ and $8.5 \%$, respectively. It can be concluded that the prediction model has good generalization ability.

\subsection{Validation of the Prediction Model}

To verify the validity of the prediction model based on the FE model data, cross-tension strength of SPR-C

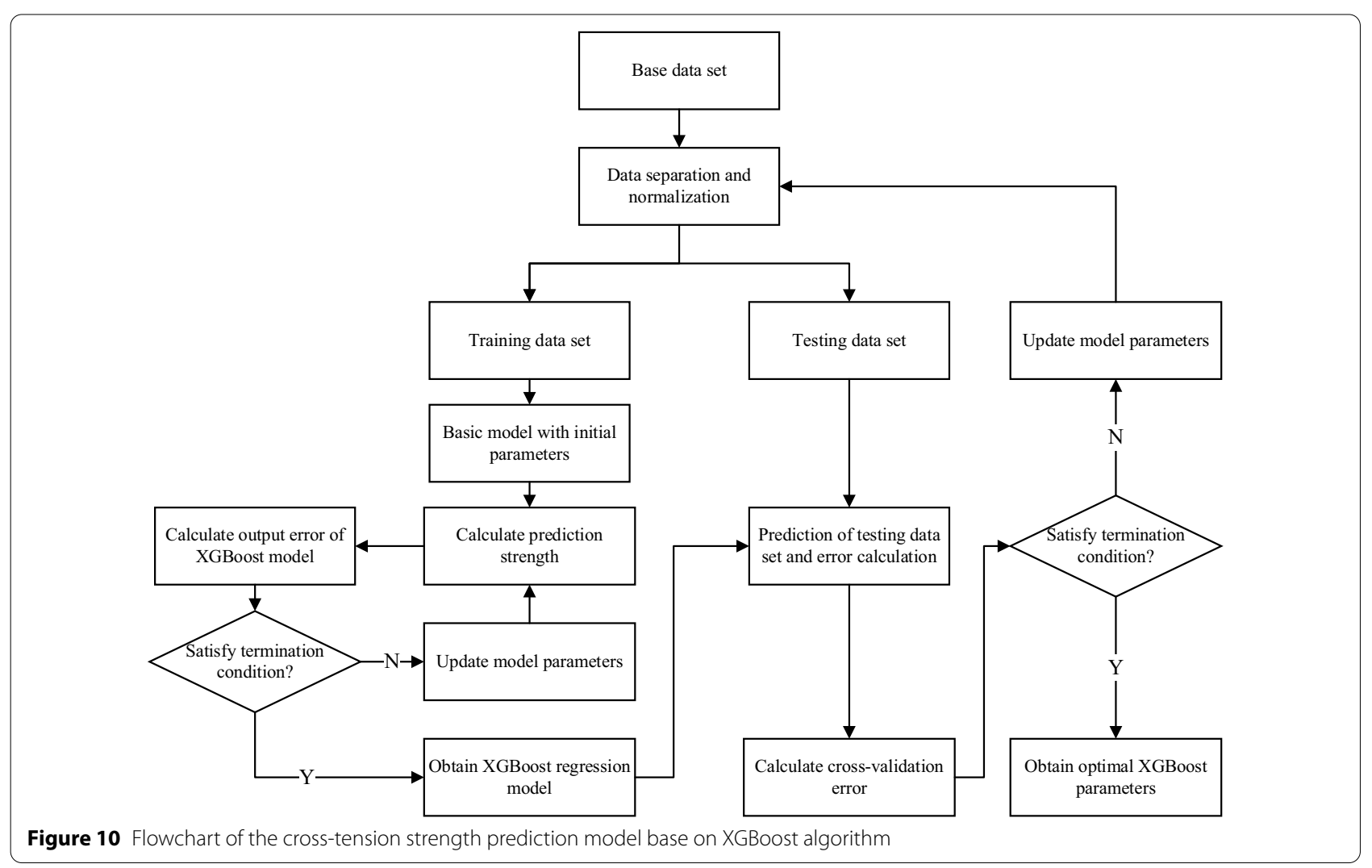




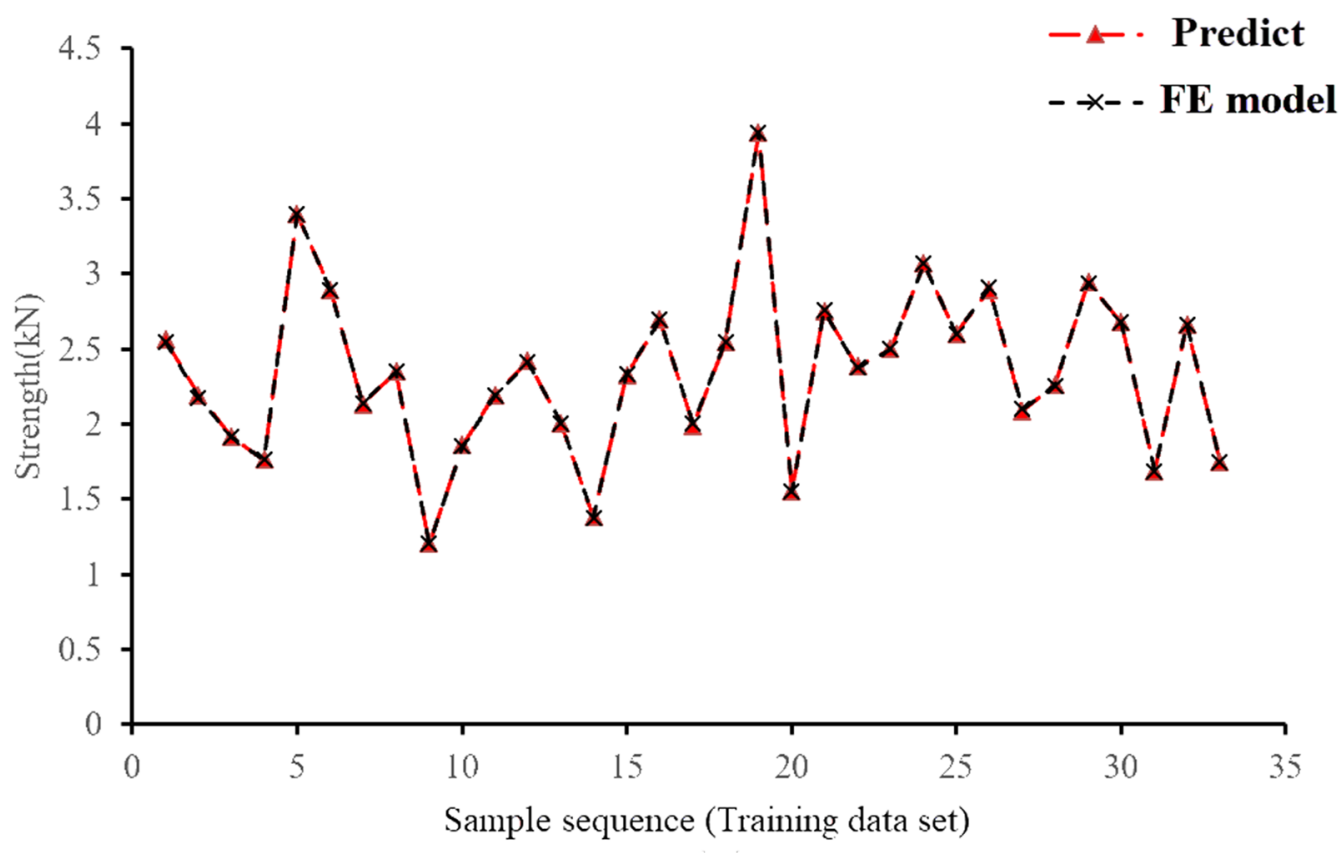

a

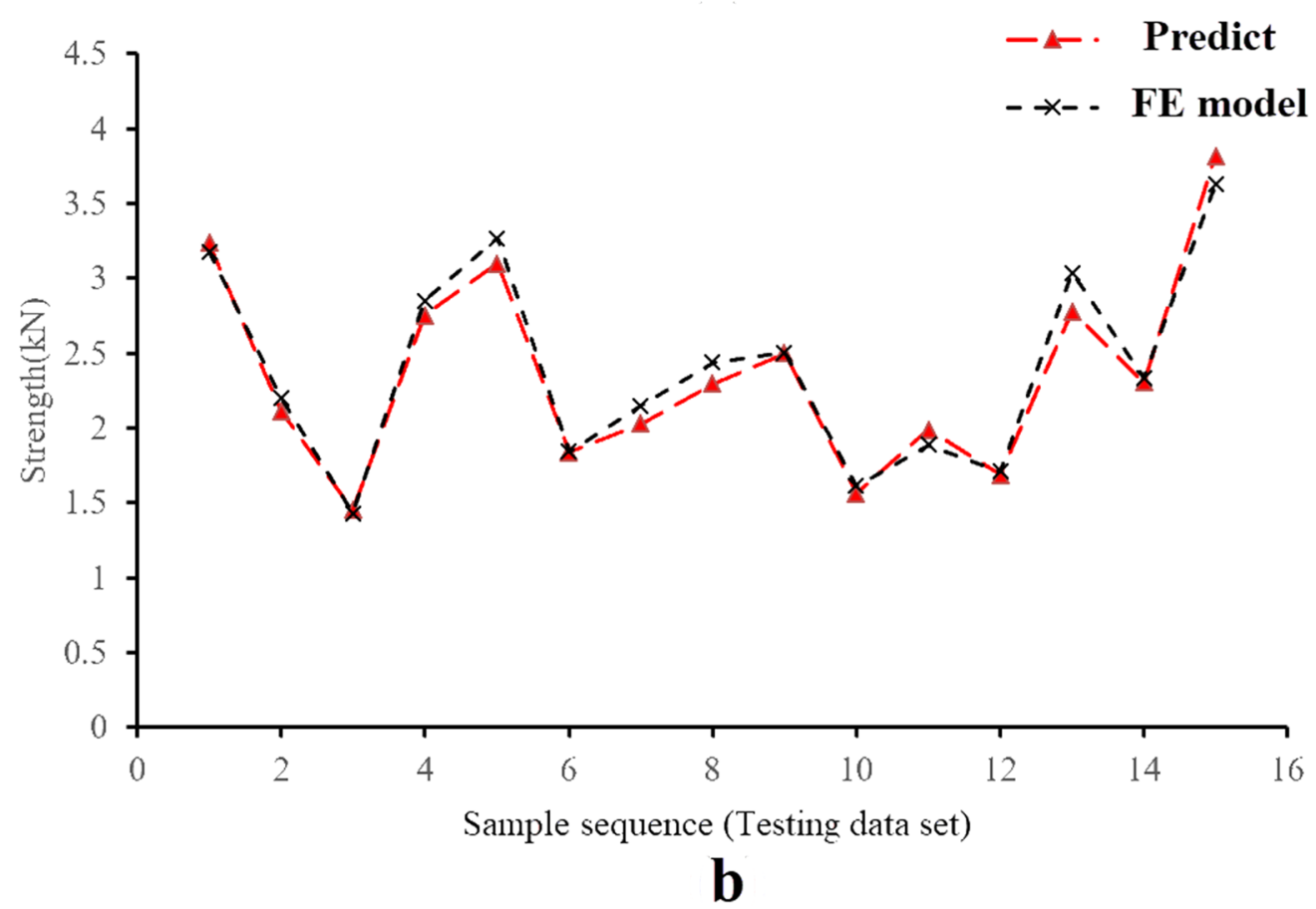

Figure 11 Comparison between prediction and input base results of cross-tension strength for (a) training data sets and (b) testing data sets

Table 6 Comparison of the cross-tension strength from experiment, FE model and the prediction model

\begin{tabular}{|c|c|c|c|}
\hline Resource & Experiment & FE model & Prediction \\
\hline Cross-tension strength (kN) & 2.52 & 2.69 & 2.71 \\
\hline Deviation (kN) & \pm 0.02 & / & / \\
\hline
\end{tabular}

joint was tested as a reference value for the prediction value of XGBoost regression model. As shown in Table 6, the averaged cross-tension strength of three joints was measured as $2.52 \mathrm{kN}$, and the cross-tension strength obtained from FE model simulation is 2.69 $\mathrm{kN}$, while the predicted strength value of SPR-C joint 
obtained from the XGBoost regression model is 2.71 $\mathrm{kN}$, and a prediction error of $7.6 \%$ is observed between experimental and predicted strengths, which indicates that the established XGBoost regression model can effectively predict the cross-tension strength of SPR joints.

Haque et al. [[10]] proposed an empirical model on the basis of characteristic force-displacement curves during SPR process, in which two critical empirical parameters depending on hardness and length of rivet were involved. Although a prediction error smaller than $8 \%$ was achieved for the cross-tension strength of SPR joint, the empirical model by Haque et al. [[10]] relied heavily on the selection of empirical parameters and a considerable number of experimental data. In contrast, the prediction method proposed in this work exhibits similar prediction accuracy through much less experiments (e.g., cross-sectional observations and cross-tension tests). In addition, FE simulation effectively enables our prediction method applicable to various experiment conditions, such as different material combinations. Another advantage of our prediction method is the capability of processing a larger amount of input data, which can be acquired through FE simulation with calibrated model rather than cost intensive experiments, to further improve the prediction accuracy.

\section{Conclusions}

In this work, a cross-tension strength prediction method based on FE model simulation and XGBoost gradient descent decision tree algorithm is proposed. A 2D FE model of SPR process was established to acquire the residual mechanical fields including plastic strain and residual stress of rivet and substrate material, which were imported into the 2D FE model of cross-tension testing of SPR joints. The cross-sectional dimensions (i.e., undercut and minimum remaining thickness) and profiles of SPR joints were used to verify the SPR process model. To acquire the required input base data sets of cross-tension strengths of SPR joints for XGBoost regression model, a 2D FE model simulation was conducted for cross-tension testing of SPR joints and validated by a good consistence between experimental and simulation results. Then, the 48 base data sets of cross-tension strengths from FE model were used to train the built XGBoost regression model to achieve the prediction for cross-tension strength of SPR joint. Using the established XGBoost regression model with optimal parameters, the cross-tension strength of the CR590(1.1 mm)/AA5754-O $(2.0 \mathrm{~mm})$ SPR joint was predicted, the experimental results showed that the prediction model could predict the cross-tension strength of the SPR joint with an error of $7.6 \%$. As a result, it is concluded that the established model based on FE model simulation and XGBoost algorithm can predict the cross-tension strength for SPR joints with a high accuracy and the XGBoost algorithm is feasible for the strength prediction of SPR joints.

The method proposed in this work exhibits obvious advantages over empirical models relying on costconsuming experiments to acquire experimental data, and it has a broad application potential and is capable to provide a guidance to predict SPR joint strength. The XGBoost algorithm, as an integrated learning algorithm, is more suitable for regression prediction on larger data sets (more than 100000 samples). Therefore, further research will focus on using the proposed method to predict cross-tension strengths of SPR joints with a larger variety of top sheet materials, rivet types and die profiles.

\section{Acknowledgements}

Not applicable.

\section{Authors' Contributions}

$J M$ and $J L$ was in charge of the whole trial; CQ finished the modeling, data analysis and wrote the manuscript; HW assisted with the manuscript checking, $J M, J L, J C$ and $L Z$ reviewed and edited the manuscript. All authors read and approved the final manuscript.

\section{Authors Information}

Jianping Lin, born in 1958, is currently a full professor at School of Mechanical Engineering, Tongji University, China. His main research interests include advanced joining and sheet forming technologies.

Chengwei Qi, born in 1997, is currently a master candidate at School of Mechanical Engineering, Tongji University, China. His research interest is advanced joining technology.

Hailang Wan, born in 1993, is currently a PhD candidate at School of Mechanical Engineering, Tongji University, China. His research interest is advanced joining technology.

Junying Min, born in 1986, is currently a full professor at School of Mechanical Engineering, Tongji University, China. His main research interests include advanced joining and sheet forming technologies.

Jiajie Chen, born in 1988, is currently an engineer at Pan Asia Technical Automobile Center, China. He received his master degree on materials science and engineering from Swiss Federal Institute of Technology in Lausa, Switzerland, in 2013.

Kai Zhang, born in 1987, is currently a PhD candidate at School of Mechanical Engineering, Tongji University, China.

Li Zhang, born in 1987, is currently an engineer at Pan Asia Technical Automobile Center, China. She received her master degree on materials science and engineering from Shanghai Jiao Tong University, China, in 2012.

\section{Funding}

Supported by National Natural Science Foundation of China (Grant No. 51805375).

\section{Competing Interests}

The authors declare no competing financial interests.

\section{Author Details}

1 School of Mechanical Engineering, Tongji University, Shanghai 201804, China. 2 Pan Asia Technical Automotive Center, Shanghai 202106, China. 
Received: 2 June 2020 Revised: 26 January 2021 Accepted: 2 March 2021 Published online: 22 March 2021

\section{References}

[1] J Min, K Zhang, S Wang, et al. Effects of rivet structure on penetration force in friction stir blind riveting of aluminum alloy sheets. Journal of Mechanical Engineering, 2020, 56(6): 159-168. (in Chinese)

[2] L Liu, Qin Yu, Z Wang, et al. Making ultrastrong steel tough by grainboundary delamination. Science, 2020: eaba9413.

[3] Y Hou, J Min, N Gu, et al. Investigation of evolving yield surfaces of dualphase steels. Journal of Material Processing Technology, 2019

[4] J Lin, Y Hou, J Min, et al. Effect of constitutive model on springback prediction of MP980 and AA6022-T4. International Journal of Material Forming, 2019, 13: 1-13

[5] K Mori, T Kato, Y Abe, et al. Plastic joining of ultra high strength steel and aluminium alloy sheets by self piercing rivet. CIRP Annals, 2006, 55(1): 283-286.

[6] K Martinsen, S J Hu, B E Carlson. Joining of dissimilar materials. CIRP Annals, 2015, 64(2): 679-699.

[7] G Michalos, S Makris, N Papakostas, et al. Automotive assembly technologies review: challenges and outlook for a flexible and adaptive approach. CIRP Journal of Manufacturing Science and Technology, 2010, 2(2): 81-91.

[8] H Jiang, C Zeng, G Li, et al. Effect of locking mode on mechanical properties and failure behavior of CFRP/Al electromagnetic riveted joint. Composite Structures, 2020: 113162.

[9] H Jiang, Y Cong, J Zhang, et al. Fatigue response of electromagnetic riveted joints with different rivet dies subjected to pull-out loading. International Journal of Fatigue, 2019, 129: 105238.1-105238.13.

[10] R Haque, Y Durandet. Strength prediction of self-pierce riveted joint in cross-tension and lap-shear. Materials \& Design, 2016, 108: 666-678.

[11] Z Xie, W Yan, CYu, et al. Tensile capacity of self-piercing rivet connections in thin-walled steel structures. Journal of Constructional Steel Research, 2018, 144: 211-220.

[12] W Yan, Z Xie, CYu, et al. Experimental investigation and design method for the shear strength of self-piercing rivet connections in thin-walled steel structures. Journal of Constructional Steel Research, 2017, 133: 231-240.

[13] Y Ma, M Lou, Y Li, et al. Effect of rivet and die on self-piercing rivetability of AA6061-T6 and mild steel CR4 of different gauges. Journal of Materials Processing Technology, 2018, 251: 282-294.

[14] CWesterberg. Finite element simulation OF crash testing of self-piercing rivet joints, peel specimen. Lund University, 2006

[15] R Porcaro, A G Hanssen, M Langseth, et al. The behavior of a self-piercing riveted connection under quasi-static loading conditions. International Journal of Solids and Structures, 2006, 43(17): 5110-5131.
[16] P O Bouchard, T Laurent, L Tollier. Numerical modeling of self-pierce riveting-From riveting process modeling down to structural analysis. Journal of Materials Processing Technology, 2008, 202(1-3): 290-300.

[17] J F C Moraes, J B Jordon, X Su, et al. Effect of process deformation history on mechanical performance of AM60B to AA6082 self-pierce riveted joints. Engineering Fracture Mechanics, 2019, 209: 92-104.

[18] H Chen, J Shen, W Chen, et al. Grinding chatter detection and identification based on BEMD and LSSVM. Chin. J. Mech. Eng., 2019, 32:1.

[19] S Shao, W Sun, RYan, et al. A deep learning approach for fault diagnosis of induction motors in manufacturing. Chinese Journal of Mechanical Engineering, 2017, 30: 1347-1356.

[20] M Fujishima, K Narimatsu, N Irino, et al. Adaptive thermal displacement compensation method based on deep learning. CIRP Journal of Manufacturing Science and Technology, 2019, 25: 22-25.

[21] M Postel, B Bugdayci, F Kuster, et al. Neural network supported inverse parameter identification for stability predictions in milling. CIRP Journal of Manufacturing Science and Technology, 2020.

[22] G Yavuz, M H Arslan, O K Baykan, et al. Shear strength predicting of FRPstrengthened RC beams by using artificial neural networks. Science and Engineering of Composite Materials, 2014, 21(2): 239-255.

[23] X Qin, S Cui, L Liu, et al. Prediction of mechanical strength based on deep learning using the scanning electron image of microscopic cemented paste backfill. Advances in Civil Engineering, 2018: 1-7.

[24] J T Liu, Z Zhao, Y Bi, et al. Prediction and compensation of force-induced deformation for a dual-machine-based riveting system using FEM and neural network. The International Journal of Advanced Manufacturing Technology, 2019, 103(9-12): 3853-3870.

[25] F Hönsch, J Domitner, C Sommitsch, et al. Numerical simulation and experimental validation of self-piercing riveting (SPR) of 6xxx aluminium alloys for automotive applications. Journal of Physics: Conference Series, 2018: 1063.

[26] Y Abe, T Kato, K Mori. Self-piercing riveting of high tensile strength steel and aluminium alloy sheets using conventional rivet and die. Journal of Materials Processing Technology, 2009, 209(8): 3914-3922.

[27] X B Zhu, X Yang, Y B Li, et al. Reinforcing cross-tension strength of adhesively bonded joints using metallic solder ball. International Journal of Adhesion \& Adhesives, 2016, 68: 263-272.

[28] M Carandente, R J Dashwood, I G Masters, et al. Improvements in numerical simulation of the SPR process using a thermo-mechanical finite element analysis. Journal of Materials Processing Technology, 2016, 236: 148-161.

[29] R Haque. Quality of self-piercing riveting (SPR) joints from cross-sectional perspective: A review. Archives of Civil and Mechanical Engineering, 2018, 18(1): 83-93.

[30] T Q Chen. XGBoost: A scalable tree boosting system. The 22nd ACM SIGKDD International Conference ACM, 2016.

\section{Submit your manuscript to a SpringerOpen ${ }^{\circ}$ journal and benefit from:}

- Convenient online submission

- Rigorous peer review

- Open access: articles freely available online

- High visibility within the field

Retaining the copyright to your article

Submit your next manuscript at springeropen.com 